\title{
APPLICATION OF VARIATION OF THE PARAMETERS METHOD FOR MICROPOLAR FLOW IN A POROUS CHANNEL
}

\author{
Osman Güngör ${ }^{1}$, Cihat Arslantürk ${ }^{2}$ \\ ${ }^{I}$ Technical Scientific Vocational School, Bayburt University \\ Bayburt, Turkey \\ ${ }^{2}$ Faculty of Engineering, Ataturk University \\ Erzurum, Turkey \\ osmangungor@bayburt.edu.tr,carslan@atauni.edu.tr
}

Received: 15 December 2019; Accepted: 24 March 2020

\begin{abstract}
This work devoted to study the injective micropolar flow in a porous channel. The flow is driven by suction or injection on the channel walls, and the micropolar model is used to characterize the working fluid. The governing nonlinear partial differential equations are reduced to the nonlinear ordinary coupled differential equations by using Berman's similarity transformation. These equations are solved for large mass transfer via variation of parameters method (VPM) which has been used effectively in the solution of nonlinear equations recently. This method has not previously been applied to a problem of micropolar flow. The results of the variation of parameters method are found to be in excellent agreement with the results of the Matlab bvp4c solver (NUM). With this validity, the effects of the some important parameters on the velocity and rotation profile of micropolar flow are discussed in detail. It can be seen that increases in the values $N_{1}$ and $N_{3}$ have different results in comparison with $N_{2}$ increasing.
\end{abstract}

MSC 2010: $35 A 15,34 L 30$

Keywords: micropolar flow, porous channel, variation of parameters method

\begin{tabular}{|llll|}
\hline Nomenclature & & \\
$c_{1}, c_{2}$ & integration constants & $W$ & Wronskian \\
$f$ & dimensionless stream function & $x, y$ & Cartesian coordinates \\
$g$ & dimensionless microrotation & \multicolumn{2}{l|}{ Greek symbols } \\
$h$ & width of channel $[\mathrm{m}]$ & $\eta$ & dimensionless independent variable \\
$j$ & micro-inertia density & $\eta$ & dummy variable \\
$L$ & linear operator & $\mu$ & dynamic viscosity $[\mathrm{kg} / \mathrm{ms}]$ \\
$N$ & microrotation/angular velocity $[1 / \mathrm{s}]$ & $\kappa$ & coupling coefficient $[\mathrm{kg} / \mathrm{ms}]$ \\
$N_{1,2,3}$ & dimensionless parameters & $\rho$ & fluid density $\left[\mathrm{kg} / \mathrm{m}^{3}\right]$ \\
$P$ & pressure & $\psi$ & stream function $\left[\mathrm{m}^{2} / \mathrm{s}\right]$ \\
$q$ & mass transfer parameter $[\mathrm{m} / \mathrm{s}]$ & $\Phi$ & dependent variable \\
$R e$ & Reynolds number & $X$ & dummy variable \\
$u, v$ & Cartesian velocity components $[\mathrm{m} / \mathrm{s}]$ & Subscripts \\
$v_{s}$ & microrotation viscosity $[\mathrm{kg} \cdot \mathrm{m} / \mathrm{s}]$ & $c$ & complementary solution \\
$v_{1,2,3,4}$ & particular solution functions & $p$ & particular solution \\
\hline
\end{tabular}




\section{Introduction}

Eringen [1] firstly introduced the concept of the micropolar fluids. It is well known that in many of the real fluids the shear behaviour cannot be characterized by Newtonian relationships and therefore, researchers have proposed diverse non-Newtonian fluid theories to explain the divergence in the behaviour of real fluids with that of Newtonian fluids. One such theory is that of micropolar fluids. The theory accounts for the internal characteristics of the substructure particles with the assumption that they are allowed to undergo rotation independent of their linear velocity. It is noticed that the theory of micropolar fluids is supposed to successfully characterize non-Newtonian behaviour of certain fluids, such as liquid crystals, liquid with polymer additives, ferro-liquids, colloidal fluids, animal blood particles, suspensions, slurries, geomorphological sediments, haematological suspensions, etc. The research area of micropolar fluids has been of great interest, mainly because the Navier-Stokes equations for Newtonian fluids cannot successfully describe the characteristics of fluid with nanoparticles [2, 3]. The equations of motion characterizing a micropolar fluid flow are non-linear in nature (as in the case of Newtonian viscous fluids) and are constituted by a coupled system of vector differential equations in velocity and rotation. The mathematical background of the micropolar fluid flow theory is presented by Lukaszewicz [4].

Most of the fluid mechanics and heat transfer problems can be transformed to an ordinary differential equation using similarity transformation, and the micropolar flow is one of them. The nonlinear differential equations arising from the presented study have been solved via a similarity transformation proposed by Berman [5], who determined an exact solution of the Navier-Stokes equations by reducing the governing partial differential equations to a nonlinear ordinary differential equation of fourth order. In the following years, the study of Berman's exact solution has attracted the attention of various researchers subsequently, i.e. Yuan [6], Robinson [7] and Zaturska et al. [8].

The present study investigates the analysis of the flow of a micropolar fluid in a porous channel, where the flow is driven by uniform mass transfer through the channel walls. The nonlinear equations in this study have been solved using a variation of the parameters method. In recent years, some researchers used several analytical/numerical methods to solve these kinds of the problems using the Optimal homotopy asymptotic method (OHAM) by Joneidi et al. [9], using the Homotopy analysis method (HAM) by Hassan and Rashidi [10], using the Homotopy analysis method (HAM) by Abdulaziz et al. [11], using the Homotopy analysis method (HAM) by Ziabakhsh and Domairry [12], using the Adomian decomposition method (ADM) by Aski et al. [13], using the Differential transform method by Mosayebidorcheh [14], using the Homotopy analysis method (HAM) by $\mathrm{Si}$ et al. [15], Homotopy perturbation method (HPM) by Sheikholeslami et al. [16], using the Lie group method by Cao et al. [17]. 
It is obvious from literature review [9-17] that various analytical and numerical approaches have been utilized so far in the analysis of micropolar flow in a permeable channel with different mathematical models. However, no research to date has been aimed at using the variation of parameters method to assess the analysis of micropolar flow in a porous channel with high mass transfer. In addition, the method of variation of parameters to evaluate in engineering problems that arising from the modeling of micropolar fluid has not been simulated previously.

The variation of parameters method (VPM) has been principally applied to solve nonhomogeneous, linear differential equations [18]. It is obvious that this method can also be used to solve nonlinear differential equations $[19,20]$. These studies have addressed general mathematical aspects of the obtained solutions. However, it is unequivocal from literature [21] that the method of variation of parameters has been used to solve nonlinear differential equations that arising in various direct and inverse heat transfer applications. Moreover, this method is a technique that is simple and relatively easy to implement for solving the nonlinear equations of complicated problems such as absorbing, and emitting non-gray planar media [22]. Arslantürk [23-25] applied this method for the thermal analysis of solid fins with different profiles and the analysis of the optimal dimensions of a space radiator. Güngör and Arslantürk [26] studied the three-dimensional problem of condensation film on an obliqued rotating disk under the steady-state condition using the variation of parameters method. Moreover, this method enables the determination of unknown variable parameters that arise from nonlinear equations where no closed form solutions are available.Therefore, it is expected that this method can be used in the solution of boundary layer equations [27] that must be solved iteratively by fulfilling a suitable boundary condition that can transform an interval problem into a finite interval problem.

The main objective of the present study is to evaluate the flow analysis of micropolar fluid through a porous channel using the variation of parameters method. With a view to evaluating the accuracy of this approach, the problem is also solved numerically using the Matlab bvp4c solver. It is observed that there is an excellent agreement between the results of compared methods.

\section{Mathematical formulation}

Let us consider the steady, incompressible, laminar micropolar fluid in a channel with porous walls (Fig. 1). The channel walls are parallel to the $x$-axis and located at $y= \pm h$, where $2 h$ is the channel width. The distance $2 h$ between the porous walls is much smaller than the width and length of the channel. Both walls have equal permeability and the micropolar fluid is uniformly injected or removed at a constant velocity $q . u$ and $v$ are the velocity component in the $x$ and $y$ directions and $N$ is microrotation, respectively. Under these assumptions, the governing equations are expressed as follows: 


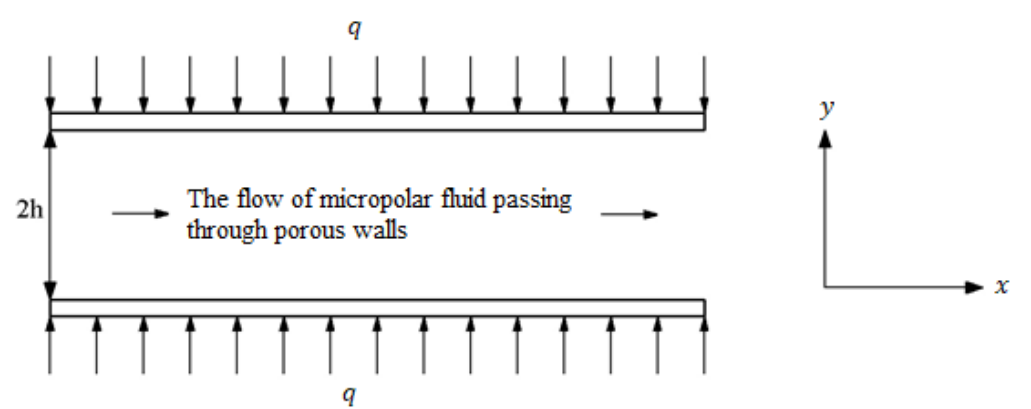

Fig. 1. Schematic diagram of the problem

$$
\begin{gathered}
\frac{\partial u}{\partial x}+\frac{\partial v}{\partial y}=0 \\
\rho\left(u \frac{\partial u}{\partial x}+v \frac{\partial u}{\partial y}\right)=-\frac{\partial P}{\partial x}+(\mu+\kappa)\left(\frac{\partial^{2} u}{\partial x^{2}}+\frac{\partial^{2} u}{\partial y^{2}}\right)+\kappa \frac{\partial N}{\partial y} \\
\rho\left(u \frac{\partial v}{\partial x}+v \frac{\partial v}{\partial y}\right)=-\frac{\partial P}{\partial y}+(\mu+\kappa)\left(\frac{\partial^{2} v}{\partial x^{2}}+\frac{\partial^{2} v}{\partial y^{2}}\right)-\kappa \frac{\partial N}{\partial x} \\
\rho\left(u \frac{\partial N}{\partial x}+v \frac{\partial N}{\partial y}\right)=-\frac{\kappa}{j}\left(2 N+\frac{\partial u}{\partial y}-\frac{\partial v}{\partial x}\right)+\left(\frac{\mu_{s}}{j}\right)\left(\frac{\partial^{2} N}{\partial x^{2}}+\frac{\partial^{2} N}{\partial y^{2}}\right)
\end{gathered}
$$

where $u$ and $v$ are the velocity components along the $x$-and $y$-axis respectively. The physical properties of the fluid are $\rho, \mu$ and $P$ which correspond to the density, the dynamic viscosity and the pressure, respectively. On the other hand, compared with Newtonian fluids, the governing equations include various parameters such as the microrotation or angular velocity $N$, the micro-inertia density $j$, a material parameter $\kappa$ and the micro rotation viscosity $\mu_{s}=\left(\mu+\frac{\kappa}{2}\right) j$. Those parameters are assumed as constants and independent. In this case, the governing equations are reduced to those presented by Berman [5].

$$
u(x, \pm h)=0, \quad v(x, \pm h)= \pm q, \quad N(x, \pm h)=-s \frac{\partial u}{\partial y} \mid x= \pm h
$$

and assuming that the flow is symmetric about $y=0$,

$$
\frac{\partial u}{\partial y}(x, 0)=v(x, 0)=0
$$

In Eq. (5); $s$ represents a boundary condition and also indicates the process to which the microelements are free to rotate close to the channel walls. For instance, while the value $s=0$ corresponds to the case where microelements close to a wall 
are unable to rotate, the value $s=0.5$ stands for weak concentrations and the disappearing of the antisymmetric part of the stress tensor.

Using Berman's similarity solution [5], the stream function and the microrotation of the micropolar fluid can be generalized by:

$$
\begin{gathered}
\psi=-q x F(\eta) \\
N=\frac{q x}{h^{2}} G(\eta)
\end{gathered}
$$

where:

$$
\eta=\frac{y}{h}, u=\frac{\partial \psi}{\partial y}=-\frac{q x}{h} F^{\prime}(\eta), \quad v=-\frac{\partial \psi}{\partial x}=q F(\eta)
$$

Furthermore, the dimensionless micropolar parameters and the Reynolds number $R e$ where for suction $R e>0$ and for injection $R e<0$ defined by:

$$
N_{1}=\frac{\kappa}{\mu}, \quad N_{2}=\frac{v_{s}}{\mu h^{2}}, \quad N_{3}=\frac{j}{h^{2}}, \quad R e=\frac{\rho q}{\mu} h
$$

where $N_{1}$ and $N_{2}$ which correspond to the coupling parameter, the spin-gradient viscosity parameter, respectively.

Substitution of Eqs. (6) and (7) into Eq. (5) yields the following system of two nonlinear differential equations:

$$
\begin{gathered}
\left(1+N_{1}\right) f^{\mathrm{IV}}-N_{1} g^{\prime \prime}-\operatorname{Re}\left(f f^{\prime \prime \prime}-f^{\prime} f^{\prime \prime}\right)=0 \\
N_{2} g^{\prime \prime}+N_{1}\left(f^{\prime \prime}-2 g\right)-N_{3} \operatorname{Re}\left(f g^{\prime}-f^{\prime} g\right)=0
\end{gathered}
$$

Subject to the boundary conditions assuming the symmetric flow in the channel:

$$
\begin{gathered}
f(0)=f^{\prime \prime}(0)=f^{\prime}(1)=0, \quad f(1)=1, \\
g(0)=g(1)=0
\end{gathered}
$$

\section{Variation of parameters method for nonlinear problems}

The method of variation of parameters is a general method that has been used to solve nonhomogeneous linear differential equations [18]. More recently, it has been known from the literature that it can also be used to solve nonlinear differential equations [19-26]. In nonlinear equations, whereas the term that disrupting homogeneity is the function of both independent and dependent variable, in linear equations this term is only the function of the independent variable. 
To illustrate the basic ideas of this method, the following general nonlinear differential equation can be considered:

$$
L \Phi=f\left(X, \Phi, \Phi^{\prime}, \Phi^{\prime \prime}, \cdots, \Phi^{(n-1)}\right)
$$

where $L$ represents a linear operator from $n$ order. The homogeneous solution of the Eq. (14) can be determined in the form:

$$
\Phi_{c}(X)=c_{1} \Phi_{c, 1}(X)+c_{2} \Phi_{c, 2}(X)+\ldots \ldots++c_{n} \Phi_{c, n}(X)
$$

where $c_{1}, c_{2}, \ldots . ., c_{n}$ are integration constants and $\Phi_{c, 1}, \Phi_{c, 2}$ and $\Phi_{c, n}$ form a fundamental set of solutions of the homogeneous equation, then a particular solution to Eq. (14) with the following form is desired:

$$
\Phi_{p}(X)=v_{1}(X) \Phi_{c, 1}(X)+v_{2}(X) \Phi_{c, 2}(X)+\cdots+v_{n}(X) \Phi_{c, n}(X)
$$

The solution procedure of the nonlinear Eq. (14) is same as the linear equations:

$$
\Phi(X)=\Phi_{c}(X)+\Phi_{p}(X)
$$

In Eq. (16), the particular solution functions can be determined given as:

$$
v_{n}(X)=\int_{0}^{X} \frac{f\left(X, \Phi, \Phi^{\prime}, \cdots, \Phi^{(n-1)}\right) W_{n}(X)}{W\left[\Phi_{c, 1}, \Phi_{c, 2}, \cdots, \Phi_{c, n}\right](X)} d X
$$

where $W$ is the Wronskian of independent solutions of the homogeneous equation

$$
W\left[\Phi_{c, 1}, \Phi_{c, 2}, \cdots, \Phi_{c, n}\right]=\left|\begin{array}{cccc}
\Phi_{c, 1} & \Phi_{c, 1} & \cdots & \Phi_{c, n} \\
\Phi_{c, 1}^{\prime} & \Phi_{c, 2}^{\prime} & \cdots & \Phi_{c, n}^{\prime} \\
\vdots & \vdots & \ddots & \vdots \\
\Phi_{c, 1}^{(n-1)} & \Phi_{c, 2}^{(n-1)} & \cdots & \Phi_{c, n}^{(n-1)}
\end{array}\right|
$$

where

$$
W_{n}(X)=(-1)^{n-k} W\left[\Phi_{c, 1}, \cdots \Phi_{c, k-1}, \Phi_{c, k+1}, \cdots \Phi_{c, n}\right](X)
$$

Because $X$ exists on both sides of Eqs. (15) and (16), an iterative process is needed to solve for $X$. Despite the fact that not a closed-form solution owing to the iteration needed, the solution can still be regarded as exact provided that $f$ is only a function of $X$ and $\Phi$ because the numerical integration required by Eq. (18a) can be performed to an arbitrary degree of accuracy. If $f$ is also a function of the derivatives of $\Phi$, finite difference equations must be used to approximate these derivatives [21]. 


\section{Numerical solution by variation of parameters method}

Eqs. (11) and (12) can be identified as follows without taking the highest order derivative term into consideration:

$$
\begin{gathered}
f^{\mathrm{Iv}}=F\left(f, f^{\prime}, g^{\prime \prime}, f^{\prime \prime}, f^{\prime \prime \prime}\right) \\
g^{\prime \prime}=G\left(f, f^{\prime}, f^{\prime \prime}, g, g^{\prime}\right)
\end{gathered}
$$

In the Eq. (19), the functions $F$ and $G$ become:

$$
\begin{gathered}
F=\left(N_{1} g^{\prime \prime}+\operatorname{Re}\left(f f^{\prime \prime \prime}-f^{\prime} f^{\prime \prime}\right)\right) /\left(1+N_{1}\right) \\
G=\left(-N_{1}\left(f^{\prime \prime}-2 g\right)+N_{3} \operatorname{Re}\left(f g^{\prime}-f^{\prime} g\right)\right) / N_{3}
\end{gathered}
$$

Equations (19) are transformed into integral equations to the form Eq. (17) according to the variation of parameters method described in the previous section. Here, this procedure will only be explained for the Eq. (19a), and only results will be presented for the other equation.

The homogeneous solution of the Eqs. (19a) are

$$
f_{c}(\eta)=c_{1} f_{c .1}(\eta)+c_{2} f_{c, 2}(\eta)+c_{3} f_{c, 3}(\eta)+c_{4} f_{c, 4}(\eta)=c_{1} \eta^{3}+c_{2} \eta^{2}+c_{3} \eta+c_{4}
$$

where $c_{1}, c_{2}$ and $c_{3}$ are integration constants and $f_{c, 1}, f_{c, 2}$ and $f_{c, 3}$ are linear independent solutions of the above homogeneous equation. It is desirable that Eq. (19a) has a particular solution as follows:

$$
f_{p}(\eta)=\eta^{3} v_{1}(\eta)+\eta^{2} v_{2}(\eta)+\eta v_{3}(\eta)+v_{4}(\eta)
$$

The general solution of the nonlinear Eq. (19a) is the sum of homogeneous and particular solutions

$$
f(\eta)=c_{1} \eta^{3}+c_{2} \eta^{2}+c_{3} \eta+c_{4}+\eta^{3} v_{1}(\eta)+\eta^{2} v_{2}(\eta)+\eta v_{3}(\eta)+v_{4}(\eta)
$$

Equations (18a)-(18c) and the particular solution functions are given by:

$$
\begin{aligned}
& v_{1}(\eta)=\frac{1}{6} \int_{0}^{\eta} F\left(f, f^{\prime}, g^{\prime \prime}, f^{\prime \prime}, f^{\prime \prime \prime}\right) d \eta \\
& v_{2}(\eta)=-\frac{1}{2} \int_{0}^{\eta} \eta F\left(f, f^{\prime}, g^{\prime \prime}, f^{\prime \prime}, f^{\prime \prime \prime}\right) d \eta \\
& v_{3}(\eta)=\frac{1}{2} \int_{0}^{\eta} \eta^{2} F\left(f, f^{\prime}, g^{\prime \prime}, f^{\prime \prime}, f^{\prime \prime \prime}\right) d \eta
\end{aligned}
$$




$$
v_{4}(\eta)=-\frac{1}{6} \int_{0}^{\eta} \eta^{3} F\left(f, f^{\prime}, g^{\prime \prime}, f^{\prime \prime}, f^{\prime \prime \prime}\right) d \eta
$$

Using the boundary conditions given by (13a), $c_{1}, c_{2}$ and $c_{3}$ constants are presented as follows:

$$
\begin{gathered}
c_{1}=-\frac{1}{6} \int_{0}^{1} F d \eta+\frac{1}{4} \int_{0}^{1} \eta F d \eta-\frac{1}{12} \int_{0}^{1} \eta^{3} F d \eta-\frac{1}{2} \\
c_{2}=c_{4}=0 \\
c_{3}=\frac{1}{4} \int_{0}^{1} \eta F d \eta-\frac{1}{2} \int_{0}^{1} \eta^{2} F d \eta+\frac{1}{4} \int_{0}^{1} \eta^{3} F d \eta+\frac{3}{2}
\end{gathered}
$$

$f$ function is obtained by substituting the functions in Eqs. (24) and $c_{1}, c_{2}$ and $c_{3}$ constants in Eq. (25) into Eq. (23):

$$
\begin{aligned}
& f(\eta)=\eta^{3}\left\{\frac{1}{6} \int_{0}^{\eta} F d \eta-\frac{1}{6} \int_{0}^{1} F d \eta+\frac{1}{4} \int_{0}^{1} \eta F d \eta-\frac{1}{12} \int_{0}^{1} \eta^{3} F d \eta-\frac{1}{2}\right\}-\frac{\eta^{2}}{2} \int_{0}^{\eta} \eta F d \eta \\
& +\eta\left\{\frac{1}{2} \int_{0}^{\eta} \eta^{2} F d \eta+\frac{1}{4} \int_{0}^{1} \eta F d \eta-\frac{1}{2} \int_{0}^{1} \eta^{2} F d \eta+\frac{1}{4} \int_{0}^{1} \eta^{3} F d \eta+\frac{3}{2}\right\}-\frac{1}{6} \int_{0}^{\eta} \eta^{3} F d \eta
\end{aligned}
$$

As indicated in Eq. (19a), $F$ is a function of the $\left(f, f^{\prime}, g^{\prime \prime}, f^{\prime \prime}, f^{\prime \prime \prime}\right)$. In this respect, because the $f$ appears on both sides and $g$ on the right side of the Eq. (26), an iterative approach is required for the solution. An initial guess for the $f$ and $g$ functions plugged into the right side of the Eq. (26).

The problem region $(0, \delta)$ is divided into the $M$ sub-region in order to obtain numerical solution of the integrals in the Eq. (26). The right sides of the equations are evaluated numerically via the trapezoidal rule provided that assigning an initial guess vector for each of the functions and a new $f$ function is calculated. This process is repeated until convergence is achieved. The derivatives in Eq. (26) are used approximately by using finite difference equations. Note that in the Eq. (26), the variation of parameters method reduces the solution of a nonlinear set of differential equations to a numerical integration solution.

The same procedure is applied to Eq. (19b) and the other function is obtained as follows:

$$
g(\eta)=\eta\left\{-\int_{0}^{1} G d \eta+\int_{0}^{1} \eta G d \eta+\int_{0}^{\eta} G d \eta\right\}-\int_{0}^{\eta} \eta G d \eta
$$




\section{Results and discussion}

In this part of the paper, the convergence and accuracy of the solution obtained via the variation of parameters method is investigated. For this purpose, the solution of the nonlinear ordinary differential equations (26) and (27) is acquired iteratively. The integrals in these equations have been solved numerically using the trapezoidal rule by dividing the problem region into the $M=500$ sub-region. Validity of the variation of parameters method is shown in Table 1. Excellent agreement between the analytical and numerical solutions is obvious in Table 1.

Table 1. The results of VPM, OHAM [9] and numerical solution for $f(\eta)$ and $g(\eta)$ when $N_{1}=N_{2}=1, N_{3}=0.1$ and $\operatorname{Re}=-1$

\begin{tabular}{|c|c|c|c|c|c|c|}
\hline \multirow{2}{*}{$\eta$} & \multicolumn{5}{|c|}{$f(\eta)$} & \multicolumn{3}{c|}{$g(\eta)$} \\
\cline { 2 - 7 } & VPM & OHAM & NUM & VPM & OHAM & NUM \\
\hline 0.0 & 0.000000 & 0.000000 & 0.000000 & 0.000000 & 0.000000 & 0.000000 \\
\hline 0.1 & 0.150798 & 0.149991 & 0.150798 & -0.040795 & -0.040103 & -0.040795 \\
\hline 0.2 & 0.298451 & 0.296953 & 0.298451 & -0.079260 & -0.077978 & -0.079260 \\
\hline 0.3 & 0.439825 & 0.437849 & 0.439825 & -0.113030 & -0.111348 & -0.113030 \\
\hline 0.4 & 0.571811 & 0.569633 & 0.571811 & -0.139680 & -0.137845 & -0.139680 \\
\hline 0.5 & 0.691341 & 0.689251 & 0.691341 & -0.156700 & -0.154969 & -0.156700 \\
\hline 0.6 & 0.795401 & 0.793657 & 0.795401 & -0.161460 & -0.160059 & -0.161460 \\
\hline 0.7 & 0.881059 & 0.879835 & 0.881059 & -0.151210 & -0.150267 & -0.151210 \\
\hline 0.8 & 0.945482 & 0.944829 & 0.945482 & -0.122990 & -0.122540 & -0.122990 \\
\hline 0.9 & 0.985973 & 0.985783 & 0.985973 & -0.073700 & -0.073611 & -0.073700 \\
\hline 1.0 & 1.000000 & 1.000000 & 1.000000 & 0.000000 & 0.000000 & 0.000000 \\
\hline
\end{tabular}

This accuracy gives us high confidence in the validity of this problem and reveals an excellent agreement of engineering accuracy. This investigation is completed by depicting the influences of some significant parameters to evaluate how these parameters affect this fluid. Moreover, the variation of the velocity $f(\eta)$ and rotation $g(\eta)$ profiles with respect to changes on independent variable $(\eta)$ is illustrated graphically.

The validity of the variation of parameters method is shown via Figures 2 and 3 . Figure 2 has been plotted in order to see the influence of velocity profile $f(\eta)$ on the values of the $\eta$ in the case of injective. As the value of $\eta$ increases the velocity profile of $f(\eta)$ increases for injection. On the other hand, Figure 3 displays the rotation profile $g(\eta)$ of the fluid with respect to changes in values of the $\eta$. It is noted that $g(\eta)$ decreases with an increase in the value of $\eta$, up to approximately 
$\eta=0.6$ and following increases with increasing $\eta$. This phenomenon can be explained as a consequence of that increment in $g(\eta)$ from suction to injection.

Figure 4 displays the effects of the Reynolds number on the velocity profile of the fluid. It is noted that the Reynolds number grows with increasing the velocity profile of $f(\eta)$ for injection. This finding is expected consequence of the injection on the channel walls. Figure 5 depicts the effects of Reynolds numbers on the rotation profile of the fluid. In a general manner, there is an increment in the rotation profile from suction to injection. It is noted that $g(\eta)$ reduces with an increment in the Reynolds number, up to roughly $\eta=0.6$ and thereafter increases with increasing Reynolds number. It is observed that with an increase in the value of the Reynolds number the point at which minimum rotation occurs does not move away from the origin of the channel.

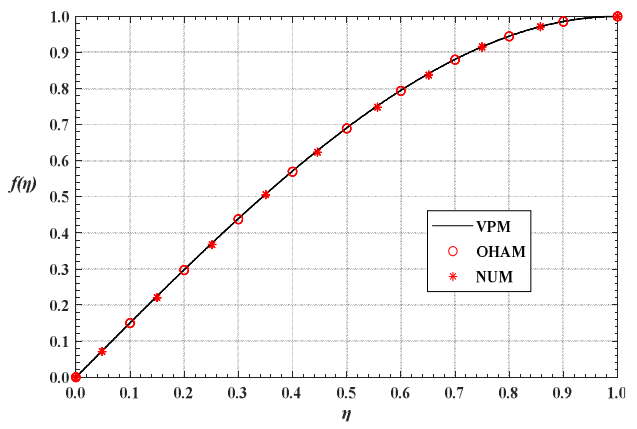

Fig. 2. The variation of the velocity profile with $\eta$ when $N_{1}=N_{2}=1$, $N_{3}=0.1$ and $\operatorname{Re}=1$

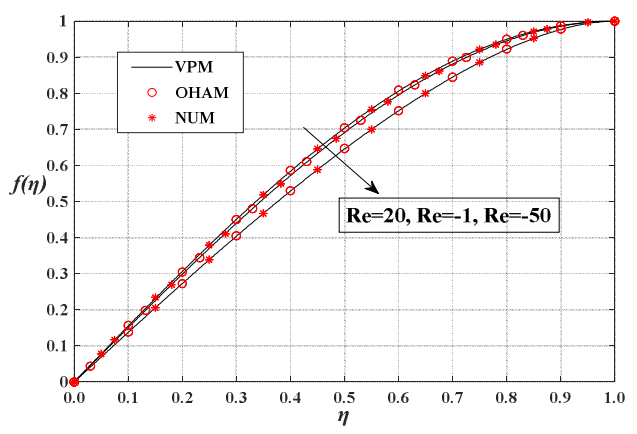

Fig. 4. Velocity profile $f(\eta)$ for different values of Reynolds number when $N_{1}=N_{2}=1$, and $N_{3}=0.1$

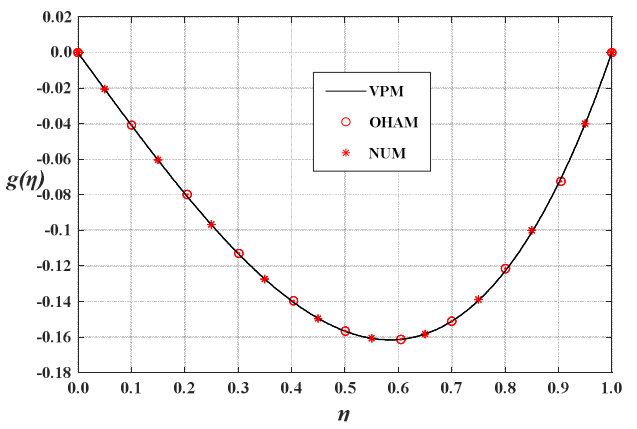

Fig. 3. The variation of the rotation profile with $\eta$ when $\boldsymbol{N}_{\mathbf{1}}=\boldsymbol{N}_{\mathbf{2}}=1$, $N_{3}=0.1$ and $\operatorname{Re}=-1$

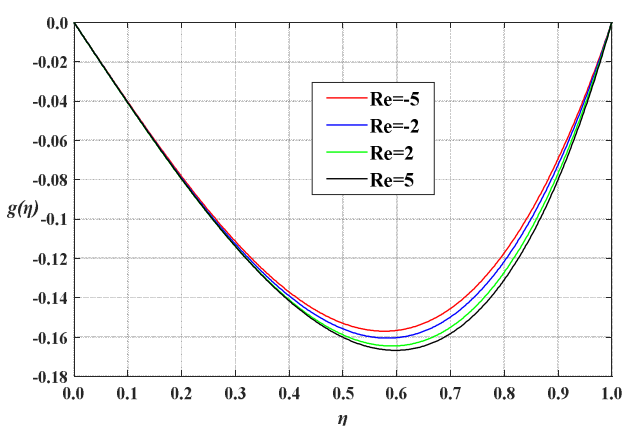

Fig. 5. Rotating profile $g(\eta)$ for different values of Reynolds number when $N_{1}=N_{2}=1$, and $N_{3}=0.1$ 


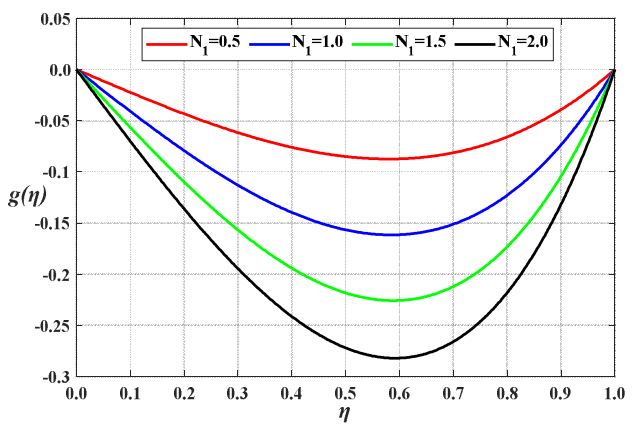

Fig. 6. Effects of different values of $N_{1}$ on rotating profile $g(\eta)$ when $N_{2}=1$, $N_{3}=0.1$ and $\operatorname{Re}=-1$

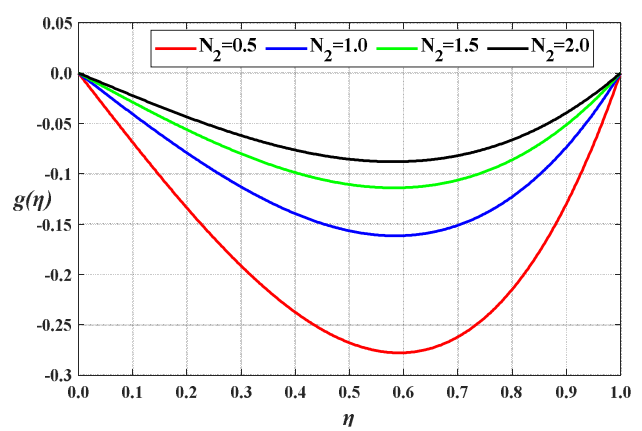

Fig. 7. Effects of different values of $N_{2}$ on rotating profile $g(\eta)$ when $N_{1}=1$, $N_{3}=0.1$ and $\operatorname{Re}=-1$

Figures 6-8 shows the effects of various values of $N_{1}, N_{2}$ and $N_{3}$ on the rotating profile. All the above results are obtained when $N_{1}$ and $N_{2}$ are fixed on 1 and $N_{3}$ is fixed on 0.1. It is observed that these parameters have no a crucial function in velocity profiles, and therefore are not remarked upon in this paper. It can be seen that increases in the values $N_{1}$ and $N_{3}$ have different results in comparison with $N_{2}$ increasing. The rotating profile $g(\eta)$ increases with increase of $N_{2}$ and decreases with increase of $N_{1}$ and $N_{3}$.

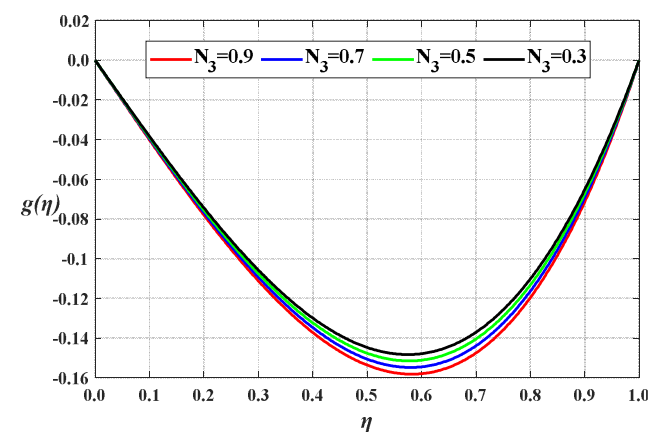

Fig. 8. Effects of different values of $N_{3}$ on rotating profile $g(\eta)$ when $N_{1}=N_{2}=1$, and $\operatorname{Re}=-1$

\section{Conclusion}

In the present study we have presented an analysis of the micropolar fluid flow in a porous channel through the channel walls. The differential form of the governing equation is formulated via a similarity transformation given by Berman [5]. The nonlinear differential equations of the micropolar flow model are computed via the variation of parameters method which has recently been successfully applied to nonlinear heat transfer problems. This approach has not been previously employed to analyze a model problem of micropolar flow. The numerical solutions 
are obtained via Matlab bvp4c solver. It is observed that there is a very excellent agreement between the solutions obtained from VPM and other methods that were compared.

After this confidence, results are demonstrated for the rotating and velocity when different values of Reynolds number Re and $N_{1}, N_{2}, N_{3}$ parameters. It is observed that an increase in the Reynolds number has various affects on velocity and rotating profile and increases in the value $N_{1}$ and $N_{3}$ have different results in comparison with $\mathrm{N}_{2}$ increasing.

\section{References}

[1] Eringen, A. (1966). Theory of micropolar fluids. Indiana University Mathematics Journal, 16(1), 1-18.

[2] Zaib, A., Haq, R.U., Sheikholeslami, M., \& Khan, U. (2020). Numerical analysis of effective Prandtl model on mixed convection flow of $\gamma \mathrm{Al}_{2} \mathrm{O}_{3}-\mathrm{H}_{2} \mathrm{O}$ nanoliquids with micropolar liquid driven through wedge. Physica Scripta, 95(3), (2020), 035005.

[3] Zaib, A., Haq, R.U., Chamkha, A.J., \& Rashidi, M.M. (2019). Impact of partial slip on mixed convective flow towards a Riga plate comprising micropolar $\mathrm{TiO}_{2}$-kerosene/water nanoparticles. International Journal of Numerical Methods for Heat \& Fluid Flow, 29(5), 1647-1662.

[4] Lukaszewicz, G. (1999). Micropolar Fluids: Theory and Applications. Basel: Birkhauser.

[5] Berman, A.S. (1953). Laminar flow in a channel with porous walls. Journal of Applied Physics, 24, 1232-1235.

[6] Yuan, S.W. (1956). Further investigation of laminar flow in channels with porous walls. Journal of Applied Physics, 27, 267-269.

[7] Robinson, W.A. (1976). The existence of multiple solutions for the laminar flow in a uniformly porous channel with suction at both walls. Journal of Engineering Mathematics, 10(1), 23-40.

[8] Zaturska, M.B., Drazin, P.G., \& Banks, W.H.H. (1988). On the flow of a viscous fluid driven along a channel by suction at porous walls. Fluid Dynamics Research, 4, 151-178.

[9] Joneidi, A.A., Ganji, D.D., \& Babaelahi, M. (2009). Micropolar flow in a porous channel with high mass transfer. International Communications in Heat and Mass Transfer, 36(10), 1082-1088.

[10] Hassan, H., \& Rashidi, M.M. (2014). An analytic solution of micropolar flow in a porous channel with mass injection using homotopy analysis method. International Journal of Numerical Methods for Heat and Fluid Flow, 24(2), 419-437.

[11] Abdulaziz, O., Noor, N.F.M., \& Hashim, I. (2009). Homotopy analysis method for fully developed MHD micropolar fluid flow between vertical porous plates. International Journal for Numerical Methods in Engineering, 78, 817-827.

[12] Ziabakhsh, Z., \& Domairry, G. (2008). Homotopy analysis solution of micro-polar flow in a porous channel with high mass transfer. Adv. Theor. Appl. Mech., 1(2), 79-94.

[13] Shakeri Aski, F., Nasirkhani, S.J., Mohammadian, E., \& Asgari, A. (2014). Application of Adomian decomposition method for micropolar flow in a porous channel. Propulsion and Power Research, 3(1), 15-21.

[14] Mosayebidorcheh, S. (2014). Analytical investigation of the micropolar flow through a porous channel with changing walls. Journal of Molecular Liquids, 196, 113-119.

[15] Si, X., Zheng, L., Lin, P., Zhang, X., \& Zhang, Y. (2013). Flow and heat transfer of a micropolar fluid in a porous channel with expanding or contracting walls. International Journal of Heat and Mass Transfer, 67, 885-895. 
[16] Sheikholeslami, M., Hatami, M., \& Ganji, D.D. (2014). Micropolar fluid flow and heat transfer in a permeable channel using analytical method. Journal of Molecular Liquids, 194, 30-36.

[17] Cao, L., Si, X., \& Zheng, L. (2015). The flow of a micropolar fluid through a porous expanding channel: A Lie group analysis. Applied Mathematics and Computation, 270, 242-250.

[18] Cengel, Y.A., \& Palm, W.J. (2012). Differential Equations for Engineers and Scientists. New York: McGraw Hill.

[19] Mohyud-din, S.T. (2013). Variation of parameters method for nonlinear diffusion equations. International Journal of Modern Applied Physics, 3(1), 48-56.

[20] Rahmatullah, \& Mohyud-Din, S.T. (2013). Variation of parameters method for nonlinear diffusion equations. International Journal of Modern Applied Physics, 3(1), 48-56.

[21] Moore, T.J. (2014). Application of Variation of Parameters to Solve Nonlinear Multimode Heat Transfer Problems. Ph.D. Thesis, Brigham Young University.

[22] Moore, T.J., \& Jones, M.R. (2014). Analysis of the conduction-radiation problem in absorbing, emitting, non-gray planar media using an exact method. Int. J. Heat and Mass Transf., 73, 804-809.

[23] Arslantürk, C. (2016). Isıl parametreleri sıcaklıkla değişen iğne kanatların, parametrelerin değişimi yöntemi ile performans analizi. Pamukkale Üniversitesi Mühendislik Bilimleri Dergisi, $22(4), 246-252$

[24] Arslantürk, C. (2018). Variation of parameters method for optimizing annular fins with variable thermal properties. Pamukkale University Journal of Engineering Sciences, 24(1), 1-7.

[25] Arslantürk, C. (2018). Optimization of space radiators accounting for variable thermal conductivity and base-to-fin radiation interaction. Proceedings of the Institution of Mechanical Engineers, Part G: Journal of Aerospace Engineering, 232(1), 121-130.

[26] Güngör, O., \& Arslantürk, C. (2019). Variation of parameters method for a three-dimensional problem of condensation film on an inclined rotating disk. Journal of Applied Mathematics and Computational Mechanics, 18(1), 15-28.

[27] Rashidi, M.M., Laraqi, N., \& Basiri Parsa, A. (2011). Analytical modeling of heat convection in magnetized micropolar fluid by using modified differential transform method. Heat TransferAsian Research, 40(3), 187-204. 